\title{
Oculofaciocardiodental syndrome: novel BCOR mutations and expression in dental cells
}

Thunyaporn Surapornsawasd, Takuya Ogawa, Michiko Tsuji and Keiji Moriyama

Journal of Human Genetics (2015) 60, 345; doi:10.1038/jhg.2015.41

Correction to: Journal of Human Genetics (2014) 59, 314-320; doi:10.1038/jhg.2014.24; published online 3 April 2014

The authors of the above paper noticed an error in publication. The scale bar; $60.0 \mathrm{~mm}$ in Figure 3a was incorrectly given as $600 \mathrm{~m}$.
This correction does not alter the results and their interpretation as discussed in the paper. The authors would like to apologize for this mistake. 